\title{
Éléments préliminaires pour construire des modèles alternatifs de croissance économique pour les pays émergents
}

\author{
Mauricio Nieto Potes ${ }^{\mathrm{a}}$ \\ "Une connaissance exacte des conditions de la croissance économique ouvrirait la porte à plus de \\ bien-être pour l'humanité et à une réduction de la misère honteuse et de la pauvreté abjecte ». \\ Douglas C. North (2007)
}

\section{INTRODUCTION}

Quand on analyse le mot «bien-être », différents points de vue apparaissent devant édifier la définition adéquate pour cette catégorie. L'école néoclassique met l'accent sur les niveaux et les taux de croissance du revenu par habitant, dont les variables déterminantes sont analysées dans un sens très restreint, comme quelque chose qui peut être versé et travaillé sur ordinateur ${ }^{1}$. Quant aux variables explicatives des changements dans le Produit Intérieur Brut par habitant (PIB/H), d'après le modèle pionnier de Solow, on inclut généralement des variables rattachées à la technologie et l'accumulation de capital humain dans leur interaction à l'accumulation du capital physique.

Bien qu'il soit aujourd'hui généralement accepté que la croissance du PIB/H est une fonction déterminée par plusieurs variables, il est aussi certain qu'on a formé un consensus où prennent principalement part des variables comme l'éducation, le stock de connaissances, les compétences développées par les travailleurs (ou efficacité du travail) et l'utilisation de la technologie, en deux mots : le taux d'accumulation $\mathrm{du}$ «stock de capital humain». On indique également la participation du «stock de capital physique », le taux par habitant et celui de l'utilisation faite par les travailleurs (efficacité). Néanmoins, des auteurs aussi rigoureux ont accepté qu'en assignant un rôle aussi grand à la technologie comme source de croissance, la théorie est aussi obligée d'assigner des rôles respectivement plus petits à tout le reste des variables ${ }^{1}$. Ainsi les modèles néoclassiques ont peu de capacité pour rendre compte de la vaste diversité que nous observons dans les taux de croissance des différents pays et dans les niveaux dissemblables de qualité de vie de leurs populations.

Selon l'hypothèse de l'égalité dans les ratios capital-travail, dans les rendements du capital et dans les salaires du modèle néoclassique, on affirme qu'elle sera atteinte grâce à la libre mobilité des biens et du capital dans les différentes économies du monde. Le modèle néoclassique revêt une impossibilité évidente pour expliquer la diversité observée entre des pays et la prévision contraire aux faits que le commerce international devrait favoriser un mouvement rapide vers l'égalité.

Sur le thème de la convergence, le courant de pensée néo-structuraliste a indiqué que les asymétries de base qui caractérisent l'économie mondiale et sa persistance au cours du temps contrastent avec la conception néoclassique de l'économie mondiale comme un contexte de relation entre pairs, comme un domaine de jeu nivelé. Dans les termes des polémiques récentes sur la croissance économique, cette vision alternative de l'économie mondiale implique que les asymétries qui la caractérisent tendent à produire des divergences de niveaux de développement ou au moins constitue un obstacle important à la convergence que supposent les théories orthodoxes de croissance économique.

Depuis la fin du $X X X^{\mathrm{e}}$ siècle, à partir de la publication de l'œuvre «Développement et Liberté » d'Amartya Sen (Prix Nobel en 1998), on a commencé à se préoccuper pour des 
variables comme le processus d'expansion des libertés réelles dont jouissent les individus en indiquant que la croissance du $\mathrm{PIB} / \mathrm{H}$, qui est la préoccupation centrale néoclassique, est évidemment un moyen très important pour développer les libertés dont jouissent les membres de la société ${ }^{2}$. Les responsables de la politique économique qui veulent favoriser la croissance du niveau de vie doivent aspirer à augmenter la capacité productive de leur pays en favorisant l'accumulation rapide de facteurs de production et en s'assurant que ceux-ci soient employés de la manière la plus efficace possible, en comprenant que l'augmentation de la capacité productive est une condition nécessaire, mais non suffisante au développement.

Quelques avancées vers l'emphase sur la qualité de vie comme variable déterminante de la croissance proviennent des travaux de la CEPAL, organisation qui a promu, il y a quelques années, les mesures au travers des recensements de population et de logement, l'indice des nécessités de base insatisfaites (NBI).

\section{On a compris qu'au-delà de la croissance du PIB par habitant, la distribution de la recette et de la satisfaction des besoins de base constituent des éléments centraux du bien-être, comme étape à partir de laquelle on pourra commencer à parler des « Libertés ».}

On a compris qu'au-delà de la croissance du PIB par habitant, la distribution de la recette et de la satisfaction des besoins de base constituent des éléments centraux du bien-être, comme étape à partir de laquelle on pourra commencer à parler des «Libertés ». La CEPAL a indiqué que les besoins de base peuvent être de caractère absolu ou relatif, les premiers étant associés à ces nécessités qui sont «absolument» nécessaires pour maintenir la vie, comme le niveau minimum de nutrition, l'attention primaire de santé, l'assistance scolaire, les caractéristiques d'habitabilité des logements et les conditions d'espace. Les secondes nécessitées (de base relatives), peuvent commencer à être considérées comme conditions du bien-être à des niveaux supérieurs de développement d'une société et réunissent des aspects comme la culture, le divertissement, les communications, l'Internet, les téléviseurs et les réfrigérateurs, biens et services qui bien qu'ils ne soient pas absolument indispensables pour survivre à partir d'un certain niveau de développement, leur absence commence à être inconcevable.

Dans les années 60 et 70, les débats sur la croissance et le développement associaient l'amélioration des éléments qualitatifs comme la santé, l'éducation et le logement au «développement », tandis que la «croissance » était mise en rapport avec l'évolution rapide du PIB par habitant, ce dernier concept pouvant inclure une distribution déficiente des revenus (Gini et indice de NBI élevé). Par la suite, les Nations Unies dans leur premier «Human Development Report» élaboré en 1990, lorsqu'une fois de plus les doctrines néo-libérales s'imposaient au monde, ont posé les analyses sur le «Concept and Measurement of Human Development », en débutant de nouvelles réflexions et systèmes de mesure du développement qui aboutiraient postérieurement aux Objectifs du Millénaire.

Sur les facteurs déterminants du développement économique, certains des modèles néoclassiques continuent le développement humain comme une variable exogène, résultat final du modèle après de longues périodes d'austérité. En réalité, la littérature néoclassique aborde très tangentiellement la possibilité que le développement humain soit un facteur qui peut être volontairement propulsé au moyen de politiques d'État et peut se traduire par des effets synergétiques positifs comme un moteur de la croissance économique. Le néo-institutionnalisme souligne que le paradigme économique en vigueur - la théorie néoclassique n'a pas été créée pour expliquer le processus de changement économique. La question centrale de la Théorie du Développement est comment et dans quelles circonstances les êtres humains créent les conditions favorables à l'existence de marchés avec de bas coûts de transaction et un bien-être matériel croissant $^{2}$ ? Dans cette dernière perspective, le fonctionnement des institutions et les bas coûts de transaction associés, deviennent des variables centrales et endogènes pour la détermination du changement et de la croissance économique. L'ac- 
ceptation et la presque «complicité » de la population avec la corruption qui a été généralisée en Amérique latine exigent des changements culturels profonds qui impliquent des modifications de la structure institutionnelle et éducationnelle commune, responsables de la génération de croyances et de perceptions partagées, unificatrices.

\section{L'acceptation et la presque « complicité » de la population avec la corruption qui a été généralisée en Amérique latine exigent des changements culturels profonds qui impliquent des modifications de la structure institutionnelle et éducationnelle commune, responsables de la génération de croyances et de perceptions partagées, unificatrices.}

Depuis la pénétration du trafic de drogues dans les différentes sphères sociales de l'Amérique latine, on a consolidé une perception unificatrice qui ne critique pas cette activité, mais l'accueille comme une activité digne d'éloges. Ainsi, une partie très significative de la population de plusieurs pays d'Amérique latine soutient des candidats et des groupes politiques clairement en rapport avec cette activité illicite. C'est sur ces soutiens et sur un taux d'actions légales et illégales que Luís Jorge Garay en 2011 attire notre attention, lui qui a développé, à la demande de la Banque Mondiale, le concept de «Capture et de Reconfiguration Cooptée de l'État » (CyRCdE).

La cooptation d'État par des groupes illégaux est consolidée principalement dans le cas de la Colombie, où « les groupes narco-paramilitaires ont expulsé la guérilla et ont obtenu un contrôle territorial dans des zones clefs pour la production et l'exportation de drogues » ou au Guatemala où les preuves indiquent qu'il y a des corps armés clandestins qui ont obtenu le contrôle de l'État; mais aussi au Mexique où les organi- sations de narcotrafiquants ont utilisé leurs ressources pour soutenir des politiciens candidats à des postes clefs, spécialement au niveau local et parfois régional. Les preuves pointent ainsi vers le fait que l'expérience d'apprentissage criminel et la consolidation de perceptions unificatrices inclinées à justifier et soutenir les activités illicites dans les trois pays mentionnés, finit par de graves processus de cooptation d'État au moyen du contrôle des processus électoraux et autres actions légales et illégales (corruptions, primes, bonifications).

\section{Depuis la pénétration du trafic de drogues dans les différentes sphères sociales de l'Amérique latine, on a consolidé une perception unificatrice qui ne critique pas cette activité, mais l'accueille comme une activité digne d'éloges. Ainsi, une partie très significative de la population de plusieurs pays d'Amérique latine soutient des candidats et des groupes politiques clairement en rapport avec cette activité illicite.}

Dans ce contexte latino-américain (et mondial), il est nécessaire d'inclure le problème de la corruption dans les modèles et d'analyser le pouvoir explicatif de cette variable sur la croissance économique.

Dans ce contexte latino-américain (et mondial), il est nécessaire d'inclure le problème de la corruption dans les modèles et d'analyser le pouvoir explicatif de cette variable sur la croissance économique.

\section{CONCEPTS THÉORIQUES ET HYPOTHÈSE DU MODÈLE}

\subsection{Antécédents}

Les premières étapes de la présente recherche ont étudié en détail les hypothèses de base utilisées par les différentes écoles de pensée économique quant à la Théorie de la Croissance et du Développement néo-institutionnaliste et postkeynésien. Les premières conclusions de cette réflexion théorique, condition préalable nécessaire à toute recherche, ont été synthétisées sous forme d'articles publiés par l'Institut de Recherches Économiques de l'Université Autonome du Mexique (UNAM) ${ }^{4}$. 
On a conclu à cette étape de la recherche, que dans les concepts : «niveau » et «qualité de vie», on devra inclure des aspects comme les libertés individuelles et des modes de vie dignes, plaisants et cultivés, c'est-à-dire un ensemble d'éléments qui sont intégralement regroupés dans les catégories : développement humain ou bien-être. On a également conclu que pour obtenir des définitions claires et objectives sur le développement, on ne peut pas utiliser de paramètres comme «le bonheur», dans lequel la Colombie a été classée dans plusieurs sondages comme vicechampion mondial, juste derrière une île paradisiaque ayant de belles plages et 200000 habitants, située dans l'Océan Pacifique. En réalité, pour donner de l'objectivité à des concepts comme: obtenir un Bien-être adéquat ou promouvoir le processus d'expansion des libertés réelles dont jouissent les individus, ceux-ci doivent être accompagnés, de la même manière que les axes stratégiques patronaux, d'objectifs quantitatifs vérifiables et d'un paquet d'indicateurs numériques dont l'évolution peut être observée. Devant l'avancée précaire de l'actuel niveau de développement, dans lequel les nécessités spirituelles, artistiques et culturelles peuvent seulement satisfaire un groupe réduit de l'humanité, les indicateurs doivent être mis en rapport, pour le moment, avec la satisfaction de nécessités primaires comme la nutrition, la santé, l'éducation, le logement et l'égalité salariale, entre autres.

Le développement exige l'élimination des principales sources de privation de la liberté : la pauvreté et la tyrannie, la pénurie d'opportunités économiques et les privations sociales systématiques, l'abandon dans lequel peuvent se trouver les services publics et l'intolérance ou l'excès d'intervention des états répressifs, il existe un principe fondamental complémentaire à celui du développement humain sur lequel on doit soutenir tout objectif de développement au $\mathrm{XXI}^{\mathrm{e}}$ siècle et qui est le respect des êtres humains, de la différence et des écosystèmes. À partir de l'acceptation de ce principe philosophique, Bioísta, conseiller stratégique, estime qu'il est nécessaire de le transformer en un des objectifs de base pour les économies en développement, ce qui à son tour exige son inclusion dans les différents processus de planification indicative. Tous ces aspects peuvent donc être analysés rigoureusement, et peuvent être enregistrés dans un ordinateur, puisque pour cela il existe des indicateurs quantitatifs précis.

L'existence de dictatures de tous types et le manque d'opportunités en rapport avec la pauvreté, le type d'éducation, la santé et le logement, sont des indicateurs qui vont en contresens d'une définition moderne et démocratique du développement.

Également, l'acceptation des objectifs de développement humain comme les «Objectifs du Millénaire » au niveau mondial présente des avantages clairs parce qu'ils sont établis comme priorité pour l'humanité dans son ensemble. La solution à la pauvreté et à la faim, la réalisation de l'enseignement primaire universel, l'égalité entre les types et l'autonomie de la femme, la réduction de la mortalité infantile, l'amélioration de la santé maternelle, la garantie de l'aptitude à soutenir des opérations prolongées de l'environnement, le combat du SIDA/VIH, le paludisme, le sarampion et d'autres maladies, dont on pensait que certaines avaient disparu de la planète, et la création d'une nouvelle association globale pour le développement.

Un avantage additionnel à cette concentration de stratégies dans les Objectifs du Millénaire, est que chacun d'eux dispose déjà des indicateurs quantitatifs qui permettent des mesures et des comparaisons de niveau mondial en spécifiant, entre autres, le pourcentage de personnes sous la ligne de pauvreté, le nombre de personnes souffrant de la famine, la scolarité, la désertion scolaire, l'analphabétisme, la morbidité et la mortalité par habitant, l'incidence de maladies endémiques et les impacts environnementaux et de réchauffement de la planète. Par conséquent, l'établissement de ces indicateurs primaires comme voie pour avancer vers le Bien-être, a l'avantage de l'objectivité face à des aspects subjectifs comme le «satisfaire», l'«utilité » et le «bonheur», qui ne peuvent pas être mesurés ni renforcés leur accroissement ou décroissement sur des périodes données et ne pas mesurer non plus les moments (analyse de «gap ») du développement qu'il reste encore à parcourir.

Or, bien qu'il existe actuellement de vastes consensus sur les objectifs sociaux et économiques du développement, on accepte «le devoir d'être » comme générique du bien-être (y aura-t-il 
quelqu'un dans le monde qui pense ainsi, qui se déclare ouvertement opposer à l'élimination de la pauvreté et de l'analphabétisme?), quand on avancera vers des politiques concrètes pour spécifier comment atteindre le Développement, les consensus ne sont déjà pas tellement clairs. À ce niveau commencent à se présenter des différences significatives tant au niveau de l' " équité » que sur la façon dont doivent être menées les politiques fiscales ou de commerce extérieur à appliquer pour l'atteindre. Apparaissent également des différences d'une plus ou moins grande portée sur le rôle que doit jouer l'État dans les politiques visant à promouvoir la croissance et dans la distribution postérieure des revenus. Comme nous l'indiquent quelques auteurs néoclassiques, une chose est d'avoir un bon rythme de croissance du " gâteau », ce qui en soi pose déjà des discussions sérieuses sur les modèles et une autre est comment on distribue cette croissance entre les différents membres de la population.

En ce qui concerne les modèles éprouvés par les économies pour l'assignation des faibles ressources et la distribution de la recette, il y a des années où on a construit des schémas alternatifs à ceux de l'économie de marché et on est arrivé à mettre en marche des pratiques de planification centrale précise qui impliquaient parfois une bureaucratie puissante et des charges fiscales inimaginables sans examiner sérieusement la possibilité que les alternatives proposées entraînent des jugements encore plus grands que ceux qu'il était attendu que produiraient les marchés ${ }^{3}$.

Un autre des consensus atteints actuellement indique que l'assignation de faibles ressources par les forces du marché représente un manque sérieux qui doit être contrôlé par les normes et les institutions, mais qui continue à être la façon la plus efficace d'effectuer cette assignation. Les plans centralisés ont démontré être un remède pire que la maladie, et le retour des pays qui ont eu une planification précise à l'assignation des ressources au moyen des lois du marché et avec une planification indicative, renforcent ces affirmations. Mais si on accepte aujourd'hui que le marché soit «le meilleur mécanisme connu pour l'assignation des ressources », il peut aussi être indiqué que dans la faute des vingt années comprises entre la chute du Mur de Berlin en 1989, la chute de Wall Street en 2008 et la crise de la zone euro en 2010, l'euphorie néo-libérale a été tellement grande que la doctrine économique a donné un saut à l'autre extrémité en lui donnant comme indique Sen, «le retour au gâteau » et a recommencé à nier de manière presque religieuse les erreurs du marché.

Durant ces années, on a généralisé l'impression que toute indication des défauts du mécanisme de marché était étrangement désuète et contraire à la culture moderne, à quelque chose comme mettre l'ancien disque de 78 tours avec une musique des années vingt.

L'impossibilité d'indiquer les erreurs du marché a été soutenue par la prédominance traditionnelle et radicale de l'école orthodoxe, mais surtout par le fait que les marchés des pays développés ont eu un bon comportement pendant les années 80 et «les joyeuses 90 », époque de l'ouverture et du Consensus de Washington. Quand, à partir du début des années 2000, les États-Unis et le reste du monde ont commencé à revivre les tristes époques des crises, les théoriciens postkeynésiens ont été écoutés avec attention et ont publié à nouveau leurs ouvrages (bien qu'il ne soit pas clair s'ils ont été lus). En effet, en 2001, la croissance du PIB des États-Unis est passée d'un excellent taux de $5 \%$ en 2000, à un $1.2 \%$ positif en 2001 , chute dont elle s'est récupérée grâce aux fortes dépenses étatiques qui ont été entreprises à la suite de l'effondrement des Tours Jumelles et à la préparation à la guerre d'Iraq. Après les trois années de récupération (2002-2004), les États-Unis ont récemment entamé en 2005 une période de ralentissement sans trêve passant de $3.3 \%$ (2005), à $2.9 \%$ (2006), puis $2.2 \%$ (2007) et enfin $1.4 \%$ (2008) jusqu'aux $2.6 \%$ négatifs de 2009. La récupération de 2010 et 2011 est arrivée à des taux de $2.6 \%$ et $2.8 \%$ respectivement, et on parle aujourd'hui d'un nouveau ralentissement du taux de croissance.

La crise due à la chute de Wall Street constitue le cadre dans lequel une fois de plus l'histoire de la pensée économique s'étudie avec une vision critique «les forces régulatrices du marché », la capacité de la «main invisible» pour amener les économies pauvres au bien-être et dans lequel récemment de manière tardive « on peut toucher le vieux disque » de la Théorie du Développement. 
Les travaux des prix Nobel d'Économie Amartya Sen, Joseph Stiglitz et Paul Krugman vont en ce sens. Espérons que durant les années d'un autre essor économique, nous n'oublierons plus les leçons de ces crises annoncées.

Finalement, quant aux hypothèses les plus générales, il est nécessaire d'indiquer que certains modèles néoclassiques sont prisonniers des approches de Arthur Lewis (l'offre illimitée de main d'œuvre...) et de Kuznets (U inversé du développement $)^{\mathrm{h}}$ dans lesquels le maintien des salaires à un bas niveau et l'augmentation de la proportion du revenu qu'apporte le capital, il augmentait à son tour l'économie et les fonds pour l'investissement et par conséquent la croissance du PIB. Bien que cela ne soit pas explicite, il était clair dans ces modèles que les bas salaires augmentaient la compétitivité des exportations, principalement celles des biens de «première nécessité » qui sont celles qui conformaient l'offre exportable des économies pauvres avec l'offre illimitée de main-d'œuvre. Dans le contexte théorique, il est indiqué que le travail s'inscrit, comme nous l'avons déjà indiqué, dans une recherche de longue portée sur l'analyse comparée du développement.

1.2 Le modèle économique appliqué en Amérique latine

\footnotetext{
Le modèle appliqué dans les économies latino-américaines pendant les trois dernières décennies (1990 à 2010) s'est inscrit dans le retour au gâteau en passant de la protection à outrance qui a été promue des années 50 aux années 80, à la prescription simple a appelé la sacro-sainte trinité du néolibéralisme : Réduction de l'État, Inflation objective et Ouverture commerciale.
}

Le modèle appliqué dans les économies latinoaméricaines pendant les trois dernières décennies (1990 à 2010) s'est inscrit dans le retour au gâteau en passant de la protection à outrance qui a été promue des années 50 aux années 80 , à la prescription simple a appelé la sacro-sainte trinité du néo-libéralisme : réduction de l'État, inflation objective et ouverture commerciale. Avec l'application de cette formule néo-libérale tridimensionnelle à partir des années 90, l'économie colombienne a commencé à présenter un faible taux de croissance moyen de son PIB (3.7\% de croissance moyenne annuelle pendant les dernières 30 années (1990-2010) face à une croissance moyenne de $5.3 \%$ entre 1950-1989.

Cette diminution dans le rythme de croissance a été principalement due à la lente dynamique de la Demande Finale Ajoutée (DFA) expliquée par une lente croissance de la capacité du marché interne pour consommer la production locale et par la faible croissance de tout ce qui a limité à son tour l'expansion de l'investissement. Dans ce contexte, le processus d'industrialisation ou de façon plus globale d'agrégation de valeur vers des biens d'une plus grande complexité technologique a été remise à plus tard en Colombie à partir des années 90 produisant une hypertrophie des secteurs de services informels de basse productivité.

La faible dynamique exportatrice et la lenteur de l'expansion du marché interne ont produit principalement ce qui à une certaine époque a été appelé le cercle vicieux du sousdéveloppement, duquel la Colombie n'a pu se soustraire, car jusqu'à aujourd'hui elle maintient $45 \%$ de sa population sous le seuil de pauvreté.

La faible dynamique exportatrice et la lenteur de l'expansion $\mathrm{du}$ marché interne ont produit principalement ce qui, à une certaine époque, a été appelé le cercle vicieux du sous-développement, duquel la Colombie n'a pu se soustraire, car jusqu'à aujourd'hui elle maintient $45 \%$ de sa population sous le seuil de pauvreté. Les indicateurs de développement social, le volume d'emploi informel et d'autres données renforcent cette affirmation.

On maintient ainsi encore aujourd'hui la tendance observée par la CEPAL depuis les années 70, dans laquelle les économies latino-américaines montrent que certains travailleurs étaient absorbés dans les secteurs de haute productivité, mais qu'une proportion généralement majoritaire était reléguée aux secteurs de basse productivité.

Les résultats du modèle économique appliqué dans la majorité des pays d'Amérique Latine à partir du Consensus de Washington, comparant le rythme 
de croissance et les indicateurs de développement humain atteignent la structure des appareils productifs résultant des pays du Sud-est asiatique comme la Corée du Sud, Taiwan, Singapour, Hong Kong et Manille. Dans ces pays en même temps qu'on a promu le développement humain, les modèles économiques ont été fortement orientés vers les niches dynamiques du marché, c'est à dire vers des produits à valeur ajoutée en technologie et connaissance, comme la production de semiconducteurs, la branche des véhicules à moteur, bateaux, ordinateurs, équipements de télécommunications et divertissement, aciers spéciaux et industrie pétrochimique, produits pour lesquels l'espace existant sur le marché mondial a été aujourd'hui fortement réduit. Ces économies à succès sont précisément celles qui ont combiné des stratégies d'agrégation de valeur en connaissance de leurs produits d'exportation avec le renforcement de leur marché interne au moyen de plans et de programmes visant à éliminer la pauvreté et l'inégalité. Ces pays ont travaillé sous la prémisse - que déjà à cette époque avait constatée la CEPAL - de la condition de simultanéité entre l'équité et la transformation productive qui à son tour a été confirmée par les réflexions d'autres économistes dans l'analyse des cas à succès des pays du Sud-est asiatique et qui grâce au Consensus néo-libéral a été remis à plus tard en Colombie.

Les économies latino-américaines qui ont maintenu des modèles de croissance traditionnels l'ont fait sous deux hypothèses erronées. Même si les salaires sont faibles et la croissance faible, c'est-àdire que le marché interne ne se développe pas par la voie d'accroissements dans la participation, il est variable dans la demande ajoutée finale, l'économie en question peut croître à des taux importants grâce à la compétitivité que ses faibles salaires accordent à ses exportations.

Dans le $\mathrm{XXI}^{\mathrm{e}}$ siècle, les pays latino-américains peuvent exporter et être compétitifs sur le marché mondial des biens de première nécessité de basses technologies, intensifs en main-d'œuvre et produits avec de bas salaires. Ces hypothèses ont pu avoir un certain impact dans la réalité à l'époque où des pays comme la Russie, l'Inde, la Chine et l'Afrique du Sud, et d'autres pays émergents de grandes populations qui fabriquent des biens de basse technologie dans des séries et des volumes importants et avec des salaires plus faibles qu'en Amérique latine, n'avaient pas fait leur apparition sur le marché mondial. Concurrencer des biens de «première nécessité » avec des pays de population importante et aux grands volumes de production s'avère extrêmement difficile parce que ceux qui arrivent sur le marché international avec du retard font face à de très fortes barrières d'entrée.

\subsection{Les hypothèses de base}

De cette manière, les options de compétitivité restant pour les pays émergents de taille intermédiaire d'Amérique latine comme la Colombie se trouvent dans les technologies à valeur ajoutée moyenne. D'abord, dans les biens de haute technologie (high tech) comme l'aéronautique, l'industrie spatiale, les microcomposants, les machines-outils, les nouveaux matériels, la robotique ou les matières plastiques d'ingénierie, pour nommer seulement quelques produits, leur développement exigerait des programmes sectoriels qui n'auraient pas possibilité du développement sur le marché local parce qu'il n'existe pas aujourd'hui de chefs d'entreprise étant sur le point de fabriquer ce type de biens. Par conséquent, il serait nécessaire de promouvoir des programmes hautement volontaristes avec des investissements en Science et Technologie et en formation de ressources humaines d'une telle ampleur que les fonds de tous les CONICyTs de la région réunis ne pourraient pas le financer. Ensuite, pour une grande quantité de biens incorporant des technologies lisses comme les chaussettes ou les chaussures en plastique, d'importants volumes de production sont nécessaires, puisqu'ils ne sont pas rémunérés si ces volumes ne sont pas atteints, sans compter qu'il existe de plus d'importantes barrières d'entrée sur le marché international.

Il reste ainsi l'option d'ajouter de la valeur aux biens de technologie moyenne qui disposent déjà d'importantes avancées sur le marché local et qui ont un fort potentiel de croissance. D'une part, il s'agit de biens d'origine agricole et d'élevage (cacao, agrumes citriques, crevette grise, viandes sur pied ou prédécoupées et leurs dérivés) qu'on exporte déjà et qui connaissent une croissance importante grâce au marché local. D'autre part, les produits provenant des nouveaux secteurs et des services de technologies intermédiaires, (dévelop- 
pement de logiciels, génération et distribution d'énergie, externalisation-BPO\&O, services de santé et postopératoires, la chaîne textileconfection unie aux vecteurs de conception, la communication et les arts graphiques).

En synthèse, les hypothèses de base de la recherche exigent de continuer à promouvoir des processus qui, sur la base de la Recherche, du Développement et de l'Innovation (R\&D\&I) ajoutent de la valeur aux biens et aux services exportés, augmentent la rémunération de leurs marques sur les marchés mondiaux et produisent sur cette base la différenciation nécessaire des produits. Enfin, il est pertinent de fortifier son marché intérieur sur la base d'une amélioration de la qualité de vie de la population et l'augmentation de son pouvoir d'achat en employant des stratégies qui diminuent significativement les taux de chômage et les indices de pauvreté.

\section{STRUCTURE DU MODÈLE ET DESCRIPTION DES VARIABLES UTILISÉES}

La revue de la littérature sur la théorie de la croissance conclut que le développement économique est une fonction poli-déterminée dépendante d'une quantité importante de variables. Elle synthétise avec une plus grande puissance explicative les déterminantes de la croissance du PIBph.

\section{Variables déterminantes pour Barro $\mathbf{R}^{6}{ }^{6}$ :}

Selon cet auteur, les variables déterminantes et fondamentales de la croissance sont nombreuses.

- La productivité de la main-d'œuvre et le taux de croissance démographique. (Variables mis en rapport: capital physique, $\mathrm{R} \& \mathrm{D}$, développement technologique, division du travail, changement des techniques, fluctuations démographiques et taille du marché).

- Le capital-humain ou, exprimé différemment, la qualification du facteur travail et son niveau d'éducation. (Variables mises en rapport: connaissance, formation, qualification compétences, habilités).

- L'espérance de vie à la naissance de la population (Variables mises en rapport: pauvreté, indigence, santé, conditions de logement et autres déterminants de la qualité de vie).

- Le niveau de dépense du gouvernement. (Variables mises en rapport : valeur ajoutée, stimulants à l'investissement, taille du marché).

- L'inflation. (Variables mises en rapport: stabilité macro-économique, stabilité des prix, politiques monétaires et fiscales).
- Le niveau de culture démocratique. (Variables mises en rapport : fonctionnement adéquat des institutions, efficacité et efficience de l'état, niveaux de corruption) ».

\section{Variables déterminantes pour North ${ }^{2}$}

- Les changements démographiques. (Variables mises en rapport : taux de croissance de la population, valeur ajoutée, taille du marché).

- Le patrimoine de connaissances. (Variables mises en rapport: productivité de la main-d'œuvre, savoir-faire des organisations, éducation, $\mathrm{R} \& \mathrm{D}$, développement technologique, spécialisation du travail, changement technique)

- Le fonctionnement des institutions. (Variables mises en rapport : culture dans le sens de North, fonctionnement des institutions, efficacité et efficience de l'état, niveaux de corruption).

Les conditions décrites par l'équation de base du modèle sont définies de la manière suivante :

\section{PIBph $=f$ \{Compétitivité de l'économie, Conditions générales de vie de la population, fonctionnement des institutions\}}

Les hypothèses à la base de ce modèle sont :

a. Les conditions de vie de la population sont une variable exogène qui augmentera et qui ne sera égalée que dans un avenir lointain, et également,

b. Le fonctionnement des institutions cesse d'être une variable exogène pour se transformer en une des principales variables endogènes. 


\subsection{La variable dépendante: Le Produit Intérieur Brut par habitant (PIBph)}

La mesure du Produit Intérieur Brut (PIB) s'est initiée en quantifiant la Valeur Brute de la Production (VBP), tous les biens et des services produits par un pays au prix du marché, c'est-àdire que le PIB est le résultat de la multiplication de la quantité de biens produits (q) par leurs prix (p) de marché : VBP=PxQ. Pour éviter la double comptabilité des produits finaux de secteurs qui constituent des facteurs de production d'autres, on soustrait les consommations intermédiaires et on obtient ainsi la valeur ajoutée par l'économie dans son ensemble. Pour arriver au Produit Intérieur Brut, on ajoute à ce chiffre le solde net des subventions et les impôts et on obtient ainsi toute la production d'une économie sur une période donnée $^{\mathrm{p}}$. Quant à ce PIB, il se divisera par le total de la population de l'économie respective, pour obtenir le PIB par habitant (ou PIB per capita), mesure qui constitue une moyenne puissante de ce que chaque personne ajoute à la production. La statistique utilisée dans cet article est celle qui présente la base de données de la CIA, dans son World Factbook (2010).

À partir de cette mesure du produit par habitant, on ne peut pas tirer de conclusions sur le bien-être ni la distribution de la recette. Donc un niveau important de production par habitant peut coïncider avec de bas niveaux de qualité de vie pour une vaste proportion de la population. L'illustration de cette affirmation peut donner le coefficient de corrélation multiple entre le PIBph et l'espérance de vie à la naissance (bon indicateur de la qualité de vie) pour 189 pays, ce fait apparaître une valeur de seulement $\mathrm{R}^{2}=26.3 \%$ avec des probabilités de coefficients très proches de zéro. On renforce ainsi la thèse qu'il n'existe pas de corrélation importante entre la croissance du PIBph et le bien-être équivalent à la production par habitant est seulement une condition nécessaire, mais pas suffisante au développement ${ }^{\mathrm{q}}$.

\subsection{Les variables explicatives du modèle}

\section{La compétitivité des économies. L'Indice de} Compétitivité Globale (ICG)

Du point de vue théorique, la compétitivité des économies devrait être rigoureusement mesurée par la fraction du marché que chacune d'elles possède, car il existe des variables «proxy » qui sont la valeur des exportations de chaque pays comme proportion de la valeur totale au niveau mondial. L'utilisation des exportations comme variable explicative «proxy » de la compétitivité a la limitation statistique de faire partie du PIB = $\{\mathrm{C}+\mathrm{G}+\mathrm{I}+(\mathrm{XM})\}$, comme élément de la balance commerciale, le PIBph étant la variable dont on prétend précisément expliquer les changements. Pour cette raison, il est plus intéressant d'utiliser l'Indice Global de Compétitivité (ICG) qui est un indicateur composé.

Pour cette raison, il est plus intéressant d'utiliser l'Indice Global de Compétitivité (ICG) qui est un indicateur composé, statistiquement indépendant du PIBph et qui s'approche à la mesure de la compétitivité des économies.

L'indice ICG est annuellement élaboré par le Forum Économique Mondial ${ }^{6}$ et il a été conceptuellement développé par Sala-i-Martin et a été appliqué pour la première fois en 2004. «L'ICG se base sur 12 piliers de compétitivité et offre une vision compréhensive des pays du monde aux différents niveaux de développement. Cet indice composé inclut des éléments comme l'inflation en l'incluant dans la variable de «Stabilité macro-économique» (macroeconomic stability), «le niveau d'éducation supérieure et la qualification », (higher education and training), la « compétitivité des affaires» (Business Competitiveness Index, en rapport avec la compétitivité des organisations), « la sophistication des opérations corporatives et de leurs directions stratégiques » (sophistication of company operations and strategy) et «la qualité de l'environnement national des affaires » (quality of the business environment national).

\section{Les conditions générales de vie. L'Indice de Développement Humain (IDH)}

À l'intérieur des statistiques qui permettent l'analyse comparée des économies du monde en ce qui concerne les variables du développement social, on trouve 1'Indice de Développement ${ }^{7}$ Humain (IDH) élaboré annuellement par le Programme des Nations Unies pour le Développement (PNUD). Cet indicateur composé combine trois 
variables : l'espérance de vie à la naissance $(\mathrm{EVN})$ variable qui comme il a été déjà indiqué, explique assez bien les conditions générales de vie de la population; le niveau éducationnel mesuré en fonction d'une combinaison du taux d'alphabétisation des adultes (pondération, deux tiers) et le taux brut d'immatriculation combinée de primaire, de secondaire et supérieure (pondération, un tiers), ce qui en fait la variable complémentaire à celle de l'Indice Global de Compétitivité qui, elle, se concentre sur l'éducation supérieure et sur la qualification des entreprises et finalement le niveau de vie mesuré par le PIB per capita (PPP en dollars) (UNDP, 2011).

Pour éviter les problèmes statistiques d'autocorrelation inclus dans les deux membres de l'équation du PIBph, on a utilisé des chiffres désagrégés de l'Indice en prenant de manière indépendante l'espérance de vie $(\mathrm{EVN})$ et l'Indice d'Éducation (EDU) comme complément à l'Indice EDU élaboré par le PNUD.

\section{L'éducation (EDU)}

L'importance de l'éducation comme élément de base dans la détermination de la croissance est importante puisqu'il se trouve à la base de la productivité et de la compétitivité. Dans les projets Recherche et Développement, il a été mis en valeur par presque tous les modèles de croissance depuis Solow. Son inclusion comme variable explicative de tout modèle ne requiert pas un plus grand soutien.

Il est nécessaire d'indiquer que dans ce domaine, l'Amérique latine a plus avancé dans l'éducation que vers d'autres indicateurs de développement social, surtout en rapport à des aspects quantitatifs (couverture) de niveau basique et intermédiaire. Dans l'éducation supérieure, comme l'ont indiqué les conclusions de la Seconde Rencontre Internationale Universia ${ }^{7}$, à laquelle ont assisté plus de mille recteurs de 23 pays : «en Amérique latine persiste une inégalité croissante en couverture et qualité de l'éducation supérieure. Seulement un jeune de la région sur trois peut aller à l'université, tandis que dans des pays développés, comme la Finlande, neuf sur 10 vont dans les salles de classe universitaires. » «Le nombre d'enfants qui vont à l'école est plus important qu'auparavant et la scolarité de la force de travail augmente progressivement. Toutefois, en ce qui concerne les principales mesures du succès (qualité, équité et efficience), les niveaux sont encore restreints et les progrès sont faibles ou inexistants ».

Ainsi, le système éducatif fait face à deux défis fondamentaux: augmenter la qualité de l'éducation à des niveaux où la couverture est déjà de $100 \%$ et augmenter la qualité et la quantité de l'éducation supérieure.

La massification à ce niveau est un des défis qui doivent être atteints en même temps que doivent être résolus les autres «objectifs du Millénaire» des Nations Unies.

\section{Le fonctionnement des institutions}

Dans la définition des variables déterminantes de la croissance économique et devant l'importance croissante de l'école de pensée néoinstitutionnaliste, il est nécessaire de s'arrêter sur l'analyse de la relation existante entre la croissance économique et le fonctionnement des institutions sociales et économiques et les coûts de transaction associés à ce fonctionnement. Les coûts de transaction sont les coûts insérés dans l'échange, augmentent ou diminuent en relation directe avec le fonctionnement efficace ou inefficace des institutions, des normes et des cultures réglementant les échanges. L'accomplissement des constitutions et des lois qui règlent les contrats, l'efficience des organismes de surveillance et le contrôle, la rigidité juridique des instances judiciaires qui garantissent que les lois soient respectées expliquent dans une large mesure les coûts de transaction.

Il s'avère évident que si les institutions législatives, juridiques ou gouvernementales d'un pays ont des niveaux d'efficience importants et que leur fonctionnement n'est pas entaché par la corruption, les coûts de transaction diminueront et par l'interaction entre les variables institutionnelles et économiques, la production et les libertés seront améliorées. Ce type de phénomènes en rapport avec le fonctionnement des institutions fait partie de la réponse de l'école néo-institutionnaliste, car elle considère qu'une des principales questions à 
résoudre est: pourquoi certaines sociétés ont atteint un degré de prospérité élevé tandis que d'autres (qui représentent la majorité de l'humanité) n'ont pas échappé à la pauvreté, à l'inégalité extrême et à la stagnation?

Ainsi, dans l'explication des différences des taux de croissance et des indicateurs du développement humain, le fonctionnement des institutions fait partie des variables de la plus grande importance pour les explications des questions centrales du développement. Devant les graves situations créées par le trafic de drogues dans différents pays d'Amérique Latine, les concepts élaborés par Garay sur la Co-Opted State Reconfiguration (CStR), a acquis la plus grande importance pour l'analyse de différents cas, non seulement en ce qui concerne les cas colombiens et mexicains, mais aussi d'autres pays latino-américains, comme le Guatemala.

La corruption et le trafic des drogues sont des éléments importants. En effet, un réseau criminel de narcotrafiquants paramilitaires été capable de manipuler les autorités administratives locales de la commune de Soledad - Atlántico en Colombie, et de dévier et s'approprier illégalement d'énormes sommes d'argent publiques appartenant au conseil municipal et étant destinées à financer des améliorations en éducation, santé et infrastructure. À Sucre, un département de Colombie, ces réseaux criminels ont développé un processus de cooptation de l'état (bottom-up process of Coopted State Reconfiguration) ayant commencé par les administrations locales et départementales et qui s'est terminé par la capture des secteurs exécutifs et législatifs les plus importants de l'état.

Il est aussi possible d'ajouter un autre exemple. Un réseau criminel international de trafic de drogues incluant Colombiens et Mexicains a créé un nouveau schéma de négociation en établissant des règles spécifiques sur les quantités, les prix et les procédures de transport pour introduire la drogue aux États-Unis. Enfin, l'organisation a établi des procédures de blanchiment d'argent effectué par des narcotrafiquants mexicains après que des Colombiens et des Guatémaltèques se soient chargés de la logistique et du transport de la drogue depuis la Colombie en passant par
l'Amérique Centrale, pour l'acheminer vers le Mexique et finalement, vers les États-Unis.

De cette dimension multinationale et de cooptation des États nationaux par des groupes hors-la-loi, découle le problème auquel fait face le développement de plusieurs pays latino-américains pas uniquement "pour des coûts de transaction très élevés, mais par la généralisation des pratiques de corruption dans toutes les institutions qui sont en marge du trafic de drogues.

\section{L'Indice de Perception de la Corruption (IPCo)}

Dans le contexte indiqué, le Modèle proposé dans cet article avance dans l'utilisation de l'Indice de Perception de la Corruption, qui est construit tous les ans par Transparency International et qui est une variable «proxy» du fonctionnement des institutions. Cet indice est appliqué dans 173 pays du monde et mesure l'appréciation des experts interrogés sur le degré de corruption qu'ont atteint les institutions des économies respectives.

Si on accepte que les «coûts de transaction» soient ceux qui dérivent de la recherche d'information, de la négociation, de la surveillance et de l'exécution de l'échange, de manière plus vaste, on peut affirmer qu'une partie importante des problèmes de croissance restreignant le développement de certains pays, dérive plus des coûts d'obtention de l'information, de l'utilisation faite par certains agents d'information privilégiée, incertitude quant aux titres de propriété, l'accomplissement ou l'inaccomplissement des contrats, la sécurité ou l'insécurité produite dans l'environnement d'investissement et de toutes les variables qui peuvent élever le prix des transactions normales, de l'inefficacité des usines ou du dysfonctionnement de l'appareil productif moderne. Cette dernière affirmation part de ce qui est posé par les néo-institutionnalistes sur le fait que « les institutions politiques, juridiques et culturelles se mettent en rapport avec les marchés au moyen des coûts de transaction »(Cuevas, 2007). Si les institutions des pouvoirs exécutifs, législatifs et judiciaires ne fonctionnent pas efficacement et correctement, ou pire s'ils sont cooptés, beaucoup d'échanges s'avèrent impossibles ou impliquent un coût énorme pour les particuliers. De cette 
manière, les efforts pour augmenter l'efficience des institutions ainsi que la lutte contre la corruption se transforment non seulement en une tâche de sauvetage des valeurs, de l'éthique et de la morale, mais une condition sine qua non pour le développement économique de tout pays ${ }^{9}$.

Il n'est pas facile de calculer ce que la corruption implique en termes économiques pour un pays en développement. Il existe néanmoins des études qui démontrent que les structures institutionnelles inefficaces et les grandes faiblesses des organismes de contrôle produisent d'importants coûts de transaction et augmentent inutilement les ressources utilisées par l'état. La corruption et la nécessité $\mathrm{du}$ secret qu'exige cette activité produisent de graves distorsions de l'information sur les marchés et élèvent entre autres beaucoup de coûts, les impôts. L'augmentation disproportionnée des impôts en ce qui concerne les taux de croissance de la production dans une économie qui présente de hauts niveaux de ce phénomène est appelée pour quelqu'un l'«activité sœur» de la corruption. De plus, la simple inefficacité (retards importants dans les démarches, conditions de rôles et certifications inutiles, timbres, études de notaire) est un autre coût important qui permet l'enrichissement illicite de quelques groupes illégaux «capteurs de revenus ». Ces recettes du processus de production et de création de richesse n'apparaissant pas, elles se transforment en un obstacle grave au développement à cause desdites distorsions de coûts et de prix et par l'augmentation disproportionnée des impôts. Cela produit ainsi le trafic de drogues, la corruption et l'inefficacité, coûts assumés par toute la société et qui se terminent en produisant de la richesse seulement pour quelquesuns. Notons que l'augmentation de la corruption augmente aussi le coût des démarches administratives et produit une multitude de contrôles qui ajoutent des processus aux démarches.

Ainsi, une partie de l'explication de pourquoi certaines économies ayant d'importantes structures productive et manufacturière ont modernisé leur secteur formel et ont en outre eu des occasions d'exporter comme c'est le cas du Mexique et de la plus grande économie de la planète (États-Unis et Canada). Ils n'ont pas encore pu atteindre de hauts taux de croissance comme celles de quelques pays exportateurs du Sud-est asiatique, cela peut s'expliquer par les coûts de transaction importants, les effets nuisibles de la corruption, les insuffisances institutionnelles et évidemment les chiffres d'exclusion importants, qui sont une restriction grave à la valeur ajoutée.

En synthèse, sur ce point on peut affirmer que l'Indice de Perception de la Corruption (IPCo) tend à une corrélation étroite entre la corruption et la pauvreté, qui se matérialise par une accumulation d'États appauvris à la fin de la classification. La corruption met des millions de personnes dans la pauvreté. Malgré la décennie d'avancées dans la définition de lois et réglementations anticorruption, les résultats que nous présentons aujourd'hui indiquent qu'il reste encore beaucoup à faire avant que nous puissions enregistrer une amélioration significative dans la vie des citoyens les plus pauvres du monde.

En effet, l'indice de Transparency International (2011) montre que les pays émergents ayant le plus de succès et auxquels on a fait référence dans cet article, occupent dans le hit-parade une position privilégiée. Des 173 économies pour lesquelles on a obtenu des données, Singapour occupe le poste numéro 1, Hong Kong le 13, l'Irlande le 14, Taiwan le 34, la Corée du Sud le 39 et le Costa Rica le 41. La Colombie, le Guatemala et le Mexique (pays fortement touchés par le trafic de drogues) occupent respectivement les places 78, 91 et 95 tandis que le Chili et l'Uruguay occupent des places de basse perception de corruption, obtenant les places 21 et 24 dans le monde et les deux meilleures places en Amérique latine. Des cinq perceptions avec un fort niveau de corruption entre les 173 pays se trouvent des économies très pauvres: le Soudan, l'Iraq, la Birmanie, l'Afghanistan et la Somalie.

Comme un exemple d'avancées de réglementation peu effective indique que les lois qui certifient les plans de développement de différentes nations proposent des actions dans ce domaine. Entre autres, le Plan de Développement de la Colombie correspondant aux années 2002-2006, cherche à augmenter la transparence et l'efficience de l'État, en considérant que le panorama actuel de l'état colombien est préoccupant. La légitimité de L'État de la Colombie a été détériorée par l'effet pervers de la corruption, le clientélisme et les relations politiques douteuses. 


\section{ESTIMATION DU MODÈLE ET CONCLUSIONS}

L'estimation du modèle a été construite sur une base de données pour une analyse en coupe transversale avec des observations de 2010 pour 193 pays enregistrés et ayant une reconnaissance internationale et appartenant aux Nations Unies. Des 193 états dont on a obtenu des données sur le PIBph et l'espérance de vie à la naissance pour 189 nations, sur la compétitivité avec leurs variables désagrégées pour 133 pays, sur le fonctionnement des institutions pour 173 pays et sur l'éducation pour 169 pays. On a évidemment pu établir ainsi, des échantillons hautement représentatifs des 193 pays reconnus pour les variables du modèle, tous les niveaux de PIBph au niveau mondial, de sorte qu'on peut effectuer les corrélations simples ainsi que les analyses de régression. De ces groupes de pays, ont été classés à l'intérieur 17 latinoaméricains. Pour les analyses qui utilisent l'information sur la compétitivité sur la recherche des impacts par des Moindres Carrés Ordinaires (MCO). L'échantillon est limité à 135 observations puisque l'Indice de Concurrence Globale (ICG) est seulement enregistré actuellement pour ce nombre d'économies.

Avec cette information, on a fait deux types d'analyses différentes destinées à trouver la relation entre les variables. En premier lieu, on a effectué les statistiques descriptives de base et l'analyse de corrélation qui a fini en démontrant, comme on pouvait s'y attendre, était donnée la réflexion effectuée, une relation très forte entre le PIBph, la compétitivité, les variables du développement humain et l'indice de corruption. En second lieu, on a effectué l'exercice de Minimum Carrés Ordinaires (MCO) pour trouver l'impact moyen de chaque indicateur sur le PIB par habitant.

\section{Analyse de Corrélation et MCO}

L'analyse de corrélation utilise les statistiques descriptives de base et les «données du pannel» pour trouver la corrélation entre les variables et les évaluer en vertu d'un test d'hypothèse.

Dans le Tableau 2, on montre la matrice de corrélation des variables utilisées, toutes des logarithmes. Les coefficients de corrélation trouvés ont été positifs et significatifs. En particulier, la première colonne du tableau 1 montre une relation, étroite, positive et très significative entre le PIBph et les quatre indicateurs utilisés. De même, avec la matrice (tableau 2). Le Graphique 1 présenté dans lequel on illustre la relation des quatre indicateurs avec la variable PIBph. Pour les quatre cas, la droite d'ajustement démontre une relation positive entre ces différentes variables ce qui permet de déduire qu'à tout niveau de signification chacune des variables est positivement reliée avec le PIB par habitant.

Dès lors pour trouver des références plus précises on a utilisé l'analyse des Moindres Carrés Ordinaires avec l'intention de trouver des estimateurs qui mesureraient l'impact moyen de chaque variable sur le PIB par habitant. On a estimé le modèle déjà cité :

$$
\begin{gathered}
P I B p h=f(I G C, E V N, E D U, I P C o) \\
P I B p h=\alpha+\beta 0 I G C+\beta 1 E V N+\beta 2 E D U+\beta 3 \\
I P C o
\end{gathered}
$$

Où :

\section{PIBph : Produit Intérieur Brut par habitant IGC : Indice Global de Compétitivité EVN : Espérance de vie à la Naissance EDU : Indice d'Éducation des Nations Unies \\ IPCo : Indice de perception de la corruption \\ Bi : $\quad$ Les coefficients respectifs.}

On a utilisé un modèle doublement logarithmique qui représenterait les élasticités pertinentes, en soustrayant l'équation de la manière suivante :

$$
\begin{gathered}
\log (P I B p h)=\log (\alpha) \\
+\beta 0 \log (I G C)-\beta 1 \\
\log (E V N)+\beta 2 \log \\
(E D U)+\beta 3 \log
\end{gathered}
$$

(IPCo)

$$
\begin{gathered}
\log (P I B p h)=-5,639+2,159 \text { IGC }+2,184 \\
E V N+0,557 E D U+1,586 \text { IPCo }
\end{gathered}
$$


Les résultats sont exposés dans le Tableau 1. Sur la base conceptuelle et théorique exposée à travers tout l'article, le modèle no 1 utilise comme variables explicatives les quatre indices ICG, EVN, EDU et IPCo, tandis que le modèle no 2 a exclu l'Indice de Compétitivité Globale (ICG) ce qui a permis d'étendre la taille de l'échantillon des économies et de tester le pouvoir explicatif des variables de développement humain et des institutionnelles.

Pour le modèle no 1, on observe que $\boldsymbol{E} \boldsymbol{V} \boldsymbol{N}$ et IPCO sont significatives à tout niveau, tandis que 1'ICG l'est à $10 \%$. L'éducation variable (EDU) apparaît comme non significative. Toutefois, en faisant un essai de signification globale (statistique F) nous voyons que ce modèle est significatif à tout niveau, ce qui indique que, dans l'ensemble, les quatre indices expliquent très bien le PIB par habitant. Dans le cas du modèle no 2 , en excluant le $\boldsymbol{I C G}$, la variable éducation est significative.

Tableau 1

Estimation du modèle

\begin{tabular}{|lcc|}
\hline & $(1)$ & $(2)$ \\
& MCO & MCO \\
VARIABLES & PIBpc & PIBpc \\
\hline \multirow{2}{*}{ ICG } & $2.159 *$ & \\
& $(1.178)$ & \\
EVN & $2.184 * * *$ & $2.734 * * *$ \\
& $(0.821)$ & $(0.771)$ \\
IPCO & $1.586^{* * *}$ & $2.041 * * *$ \\
& $(0.320)$ & $(0.204)$ \\
EDU & 0.557 & $0.768 *$ \\
& $(0.419)$ & $(0.407)$ \\
Constante & $-5.639 *$ & $-5.469 *$ \\
& $(3.249)$ & $(3.277)$ \\
& & \\
Observaciones & 135 & 135 \\
R-cuadrado & 0.717 & 0.709 \\
F & 82.16 & 106.5 \\
\hline Errores estándar en & paréntesis & \\
$* * *$ p $<0.01, * * \mathrm{p}<0.05, * \mathrm{p}<0.1$ & \\
\hline
\end{tabular}

Tableau 2

Matrice de Corrélation des variables utilisées

\begin{tabular}{|r|r|r|r|r|r|}
\hline & \multicolumn{1}{|c|}{ Inpibph } & logevn & \multicolumn{1}{l|}{ logicg } & logipco & logedu \\
\hline Inpibph & 1 & & & & \\
\hline logevn & $0.6773^{*}$ & 1 & & & \\
\hline logicg & $0.8018^{*}$ & $0.7268^{*}$ & 1 & & \\
\hline logipco & $0.7915^{*}$ & $0.5721^{*}$ & $0.8548^{*}$ & 1 & \\
\hline logedu & $0.5979^{*}$ & $0.7085^{*}$ & $0.6580^{*}$ & $0.4988^{*}$ & 1 \\
\hline * Nivel de significancia $\alpha=0.001$ & \\
\hline
\end{tabular}

En ce qui concerne les coefficients, tous sont exprimés en termes d'élasticités, c'est-à-dire que pour le cas de l'Indice de Perception de la Corruption (IPCo) par exemple :

$$
\delta \text { PIBph / } \delta \text { IPCo }=1,586
$$

\section{Ainsi, une diminution de $1 \%$ des niveaux de corruption d'une économie peut conduire à des augmentations de $1.5 \%$ du PIB par habitant. Autrement dit, si le niveau de corruption de l'économie diminue de $1 \%$, le PIBph augmenterait de $1.5 \%$.}

Ainsi, une diminution de $1 \%$ des niveaux de corruption d'une économie peut conduire à des augmentations de $1.5 \%$ du PIB par habitant. Autrement dit, si le niveau de corruption de l'économie diminue de $1 \%$, le PIBph augmenterait de $1.5 \%$.

\section{La lutte contre la corruption n'est pas seulement un élément de défense de l'éthique et des valeurs, mais la tentative de dépasser un obstacle sérieux à la croissance et au développement économique.}

Le comportement de ce chiffre réaffirme l'approche faite dans le contexte de la réflexion néoinstitutionnaliste et également l'approche de Transparency International, selon laquelle la lutte contre la corruption n'est pas seulement un élément de défense de l'éthique et des valeurs, mais la tentative de dépasser un obstacle sérieux à la croissance et au développement économique. 
D'autre part, l'élasticité du $\boldsymbol{P I B p h}$, en ce qui concerne l'espérance de vie à la naissance $(\boldsymbol{E} \boldsymbol{V N})$ est le coefficient qui fait apparaître le plus grand impact sur le PIBph (2.1\%), en propulsant de manière robuste la thèse qui a été expliquée au long de cet article, dont l'amélioration de la qualité de vie des populations des économies du monde améliore simultanément l'exercice de la production de manière plus que proportionnelle vu les effets synergétiques que le bien-être provoque sur la variable. Modification de l'Espérance de Vie à la Naissance $(\boldsymbol{E} \boldsymbol{V} \boldsymbol{N})$ de $1 \%$.

Les modifications de l'espérance de vie à la naissance $(\boldsymbol{E} \boldsymbol{V} \boldsymbol{N})$ de $1 \%$ conduiraient à des hausses du rythme de croissance du PIBph de $2.1 \%$, qui est le résultat qui attire significativement l'attention sur la capacité synergétique que peuvent arriver à avoir les améliorations sur la qualité de vie sur la croissance économique.

La relation établie entre le $\boldsymbol{P I B p h}$ et l'éducation présente l'élasticité la plus faible $(0.5 \%)$ par l'écart existant entre l'investissement et ses impacts sur le $\boldsymbol{P I B}$. Additionnellement, cette analyse étant de coupe transversale, elle ne peut pas recueillir les effets des processus générationnels éducatifs. Les impacts de l'éducation doivent être préférablement calculés avec des séries de temps, puisque l'éducation n'a pas un impact sur le PIBph durant une année et son effet, sans doute très positif, est observé à moyen et long terme dans les économies. Les investissements qui en 2010 sont faits en éducation dans les différents pays seront seulement reflétés dans une coupe transversale dans plusieurs années. Également, dans la mesure où la majorité des pays de la planète sont très pauvres et ces derniers ont seulement commencé récemment à effectuer des efforts en éducation.

En analysant le graphique 1 il a été décidé d'exclure des interactions au carré, puisque la relation entre les variables est donnée d'une manière linéaire et il n'y a pas de concavités qui affectent l'estimation. On a écarté tout problème d'autocorrélation et de multicolinealité puisqu'on utilisait une coupe transversale avec une seule période de temps et de même en utilisant des indices qui ne sont pas des combinaisons d'autres vecteurs explicatifs. Finalement, on a calculé des erreurs robustes pour éviter des problèmes d'hétéroscédasticité.

Finalement, on a trouvé que les signes trouvés étant positifs, ceux-ci coïncident avec ceux attendus et fortifient ce qui est exposé par l'analyse de corrélation.

\section{Graphique 1}
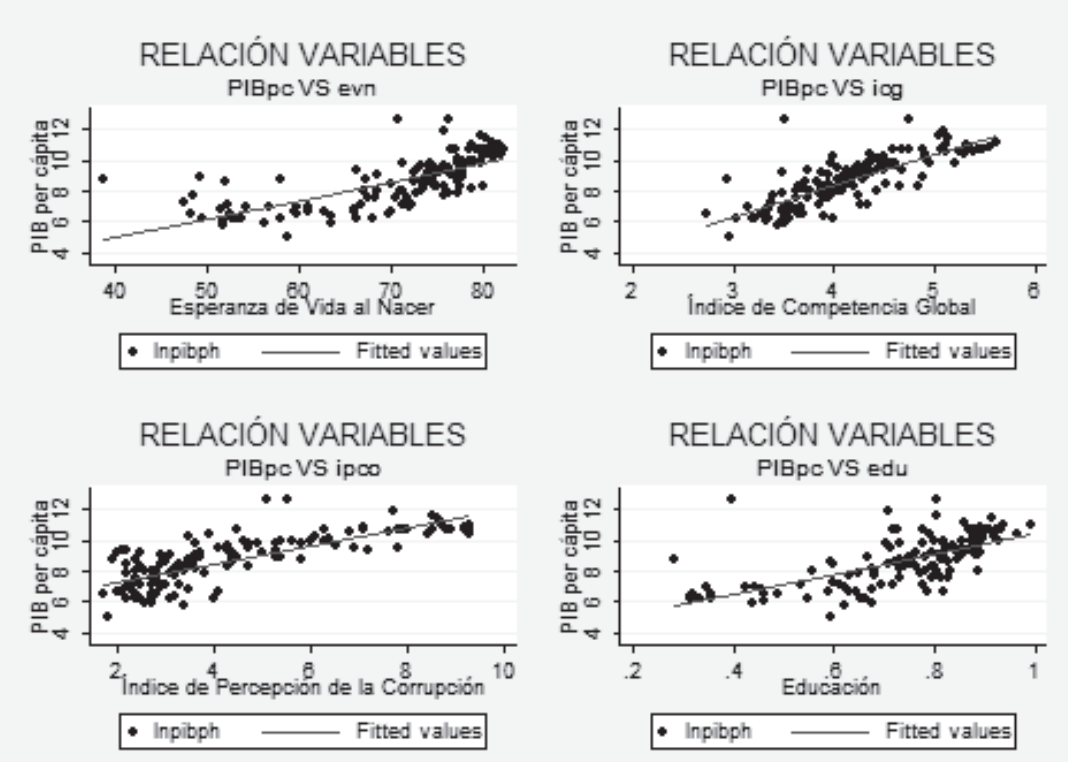

Organisations et territoires (2013) 


\section{BIBLIOGRAPHIE}

${ }^{1}$ Lucas Jr. Robert E., (2005). «Lecturas sobre crecimiento económico ». Bogotá, Economía, Universidad de los Andes, Grupo Editorial Norma.

${ }^{2}$ North Douglas, (2007). «Para entender el proceso de cambio económico». Ed. Norma, Bogotá, Colombia.

${ }^{3}$ Sen Amartya, (2009). « Desarrollo y Libertad ». Ed. Planeta. Barcelona, España. Colombia, Febrero.

${ }^{4}$ Nieto Potes Mauricio, (2009). «Apuntes alrededor de los supuestos neoclásicos de los modelos de crecimiento ». Revista Problemas del Desarrollo, Instituto de investigaciones económicas, Universidad Autónoma de México, Vol. 40, Número 159. Octubre-Diciembre.

${ }^{5}$ Max Neef Manfred, (2007). «Bienestar, Bienser y Bioísmo». Conferencia en el foro: "Desarrollo humano como estrategia para el éxito empresarial. Del Bienestar al Bienser ». Bogotá, Confamiliar, Afidro, Octubre.

${ }^{6}$ World Economic Forum - WEF -, (2011). «The Global Competitiveness Report 2010-2011». http://gcr.weforum.org/gcr2010/

${ }^{7}$ Universia, (2010). "Segundo Encuentro Internacional" Periódico La Jornada, Lunes 31 de mayo, p. 42. Guadalajara, México. http://www.jornada.unam.mx/ 2010/05/31/sociedad/042n1soc.

${ }^{8}$ Garay Luís Jorge, Salcedo-Alvarran Eduardo, (2011). «Drug trafficking. Corruption and States. How Illicit Networks Reconfigure Institutions in Colombia, Guatemala and Mexico ». http://esalbaran.com/Intro_DTCS(2011).pdf.

${ }^{9}$ Cuevas, (2007). «Teorías económicas del mercado ». Ed. 2da. Universidad Externado de Colombia. Bogotá, Colombia.

${ }^{10}$ Transparency International, (2011). «Corruption perception index, $2010 »$. http://www. Transparency.org/policy_research/surveys_indices/cpi/2010.

\section{Notes}

${ }^{a}$ Enseignant-chercheur : Université EAN - Université du Québec à Chicoutimi - UQAC, Économiste, Université National de la Colombia. 